\title{
Inflammation Research: modern design, more quality science
}

\author{
Michael J. Parnham
}

(C) Springer Basel AG 2010

Inflammation Research enters the New Year with a fresh face. Behind us lies a period of continual improvement and adaptation. This has been driven, on the one hand, by our goal to further enhance the scientific quality of the journal and on the other by process changes arising from the full integration of the publisher, as Springer Basel AG, into the Springer Science + Business Media organization.

The editorial team has carried out an extensive revision of the instructions for authors of manuscript submissions to the journal, sharpening the requirements, particularly for clinical studies, and focussing the scope. These revised instructions are now available on the journal website (http://www.springer.com/11). Authors and readers will have observed that the standards have been raised. We have also expanded the types of article which may be submitted, by encouraging the submission of articles on the history of inflammation. Review articles and book reviews continue to be welcomed. The ever-increasing number of manuscript submissions and the sustained rise in Impact Factor bear witness to the growing international recognition of the journal.

We have also enhanced integration of the journal with the societies which use Inflammation Research as their official publication organ. Abstracts from meetings of the International Association of Inflammation Societies (IAIS) and the European Histamine Research Society (EHRS) have been adapted to meet the stricter demands of electronic publishing and the members of the societies now have greater access to archival publications. Society members are encouraged to make use of your journal and submit manuscripts of all types.

With the full incorporation of the journal into the Springer organization, processing of accepted manuscripts is now faster and more efficient. Articles are rapidly published online and the number of print articles that can be published per issue has become more flexible. The open access to the journal that was temporarily provided during the month of November will undoubtedly have raised readers' interest. The complete revision of the SpringerLink website (springerlink.com) has enhanced access to the publisher's journals in general and the Inflammation Research website has profited from this process.

In the final step of the transformation, the layout and style of the journal has been brought into line with Springer journals in general. Readers will have become familiar with the Springer article layout over the last year and now the printed form of the journal has been refreshed with revised information for authors and subscribers and a modern brighter cover. This new design reflects our response to changing conditions and our ongoing efforts to publish the highest quality inflammation research in the journal. 